\title{
Tratamento multidisciplinar na reabilitação de pacientes portadores de fissura de lábio e/ou palato
}

\author{
Multidisciplinary treatment in patients rehabilitation with cleft palate and/or lip
}

MORETTO, Marcelo Juliano; FERNANDES, Samuel Lucas²; LEMES, Andressa Teixeira de Andrade ${ }^{3}$; FERREIRA, Eryca Silva ${ }^{3}$; SALES, Carmem Franscyelle Rosa ${ }^{4}$

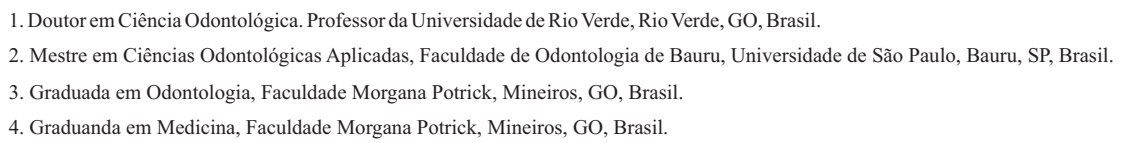

\section{RESUMO}

Este estudo teve como objetivo avaliar o conhecimento dos acadêmicos dos cursos de Enfermagem, Medicina, Odontologia e Psicologia da Faculdade Morgana Potrich sobre o tratamento multidisciplinar ao portador de fissura de lábio e/ou palato, e a necessidade ou não da abordagem do assunto dentro do referido curso. Foi realizado um estudo transversal descritivo desenvolvido por meio de um questionário semiestruturado aplicado a 200 acadêmicos da instituição, sendo selecionados 50 alunos de cada um dos cursos em estudo. Como resultados observamos uma alteração significativa no conhecimento e na abordagem dos pacientes de acordo com cada curso. Houve grande interesse pela maioria dos graduandos em obter mais informações sobre as fissuras de lábio e/ou palato, sendo que todos os acadêmicos, independente do curso relataram a importância do atendimento multiprofissional.

Palavras-chave: Fenda labial. Fissura palatina. Anormalidades congênitas.

\begin{abstract}
This study aimed to assess the knowledge of academics of Nursing, Medicine, Dentistry and Psychology Faculty Morgana Potrich on the multidisciplinary treatment to cleft lip and / or palate, and the need or not to approach the subject within that course. It conducted a cross sectional study by means of a semi-structured questionnaire administered to 200 students of the institution, being selected 50 students for each course of study. Thus, we observe a significant change in knowledge and approach according to each course. There was great interest by most of the students in learning more about cleft lip and/or palate, and all students, regardless of the course reported the importance of multidisciplinary care.
\end{abstract}

Keywords: Cleft lip. Cleft palate. Congenital abnormalities. 


\section{INTRODUÇÃO}

As fendas labiopalatais constituem uma das mais comuns malformações congênitas que acometem a face, podendo afetar tanto lábio como palato, sendo resultante de uma malformação na fusão do lábio ou palato durante a fase intrauterina1. A etiologia não é bem definida, entretanto, existem estudos que apontam os fatores ambientais e genéticos como determinantes. Os fatores ambientais estão ligados principalmente à dieta materna, suplementação vitamínica, álcool, fumo e drogas ${ }^{2-3}$. O fator genético está relacionado à hereditariedade4. As fendas labiopalatais ocorrem entre a sexta e a décima semana de gestação, sendo sua incidência de 1 a cada 650 crianças nascidas, é mais comum do lado esquerdo da face e no sexo masculino 5 .

A presença de fissuras labiopalatais aumenta a probabilidade de doenças bucais, principalmente a cárie dentaria e doenças periodontais, devido à dificuldade de higienização em algumas áreas da boca ${ }^{6}$. Além de doenças bucais, é muito comum apresentarem outras anomalias, entre elas destacam-se as agenesias, dentes supranumerários, microdontias e maloclusões, que contribuem para uma estética e fala deficiente ${ }^{7-10}$. Os portadores dessa anomalia podem apresentar diversas dificuldades e complicações simples pela ausência da integridade estrutural e anatômica, entre elas são a audição, sucção, deglutição, mastigação, fonação e respiração ${ }^{11-13}$.

A classificação mais utilizada é a classificação de Spina, por ser simples e objetiva, tendo o forame incisivo como ponto de referência. Pode ser classificada em: préforame incisiva completa, pré- forame incisiva incompleta, pós-forame incisiva completa, pós-forame incisiva incompleta e a trans forame. Todas elas podem ser uni ou bilateral, apresentando dos lados direito e esquerdo ${ }^{1,5}$.

A alimentação e a higienização são problemas característicos que necessitam de conhecimento teórico e treinamento prático ${ }^{6}$. Uma criança portadora de fissura labiopalatal necessita de cuidados especiais e deverá ter uma atenção especial para manutenção da saúde geral para que seu peso esteja ideal para a primeira correção cirúrgica, que ocorre por volta dos 3 anos de idade, pois se houver comprometimento sistêmico, este paciente deverá esperar para proceder a realização do procedimento cirúrgico ${ }^{12,14}$.

Durante a gestação, os pais sentem-se ansiosos enquanto aguardam o nascimento do bebê, o que causa grandes expectativas e emoções aliadas à ansiedade de ver o rosto da criança. Sonham e planejam como será a chegada do bebê, entretanto em casos de fissuras labiopalatais, os pais podem ser surpreendidos, o que poderá ocasionar desconforto emocional, tristeza ou até mesmo sentimento de negação, levando ao cancelamento da idealização feita do filho e imaginação de grande parte dos planos e anseios, fazendo com que os pais se culpem pela impossibilidade de gerar um filho perfeito. Os pais apesar de também se tornarem vítimas da situação, necessitam e devem procurar acompanhamento multidisciplinar para o bebê, acompanhamento este que refletirá de forma lenta, mas eficaz na reabilitação da criança $a^{15-16}$.

As fissuras labiopalatais propiciam uma grande diversidade de problemas aos portadores da malformação, incluindo a saúde sistêmica, bucal e psicológica ${ }^{17-18}$. Vários profissionais são responsáveis pelo tratamento dos pacientes fissurados, visto a grande variabilidade de problemas envolvidos como médicos, enfermeiros, odontólogos, nutricionistas, fonoaudiólogos, psicólogos e assistentes sociais que deverão compor uma equipe multidisciplinar ${ }^{19}$. As equipes envolvidas no tratamento devem possuir características fundamentais como a interação, colaboração, profissionalismo, cooperação e responsabilidade, portanto os profissionais envolvidos possuem grande responsabilidade, tanto com o paciente como com a família, uma vez que os familiares também necessitam de cuidados e atenção para que haja maiores e melhores informações relacionadas à reabilitação do paciente $\mathrm{e}^{20-22}$.

Assim, torna-se indispensável o conhecimento sobre as responsabilidades dos profissionais envolvidos na reabilitação de pacientes portadores de fissura labial e ou palatal e a avaliação do conhecimento adquirido durante o curso de graduação dos alunos da Faculdade Morgana Potrich sobre a necessidade do tratamento multidisciplinar.

\section{MATERIAL E MÉTODOS}

O estudo denominado "Conhecimento dos alunos de Odontologia sobre o uso da sedação consciente com óxido nitroso" foi registrado e aprovado no comitê de ética em pesquisa sob o no CAAE: 58212316.5.0000.5428.

Foi realizado um estudo transversal descritivo, realizado por meio da aplicação de questionário em sala de aula com tempo cronometrado e sem que houvesse comunicação entre os alunos, após a assinatura do termo de consentimento livre e esclarecido.

Para a coleta de dados utilizou-se um questionário semiestruturado, elaborado pelos pesquisadores, abordando dados da amostra assim como informações sobre o atendimento multidisciplinar na reabilitação de pacientes portadores de fissuras de lábio e/ou palato, aplicado aos alunos dos cursos de Enfemagem, Medicina, Odontologia, e Psicologia da Faculdade Morgana Potrich (FAMP), Mineiros - GO.

A amostra foi composta por 200 alunos devidamente matriculados nos cursos de Enfermagem, Medicina, Odontologia e Psicologia da FAMP, que concordaram com os termos da pesquisa e assinaram o termo de 
consentimento livre e esclarecido, foram selecionados aleatoriamente 50 alunos a partir do terceiro período de cada um dos cursos do estudo.

\section{RESULTADOS}

Para avaliação do conhecimento dos acadêmicos da FAMP foi elaborado um questionário sobre o tratamento multidisciplinar para os portadores de fissuras de lábio e/ou palato aplicado aos acadêmicos dos cursos de enfermagem, medicina, odontologia e psicologia, sendo selecionados 50 acadêmicos de cada um dos cursos em estudo, em um total de 200 alunos, tendo como critério básico que já houvesse concluído ao menos o terceiro semestre do respectivo curso de graduação.

Os acadêmicos foram questionados sobre o conhecimento sobre as fissuras labiais e palatinas, nos cursos de medicina e odontologia $98 \%$ dos alunos relataram terem ouvido falar da anomalia contra $72 \%$ da enfermagem e $52 \%$ do curso de psicologia (Figura 1).
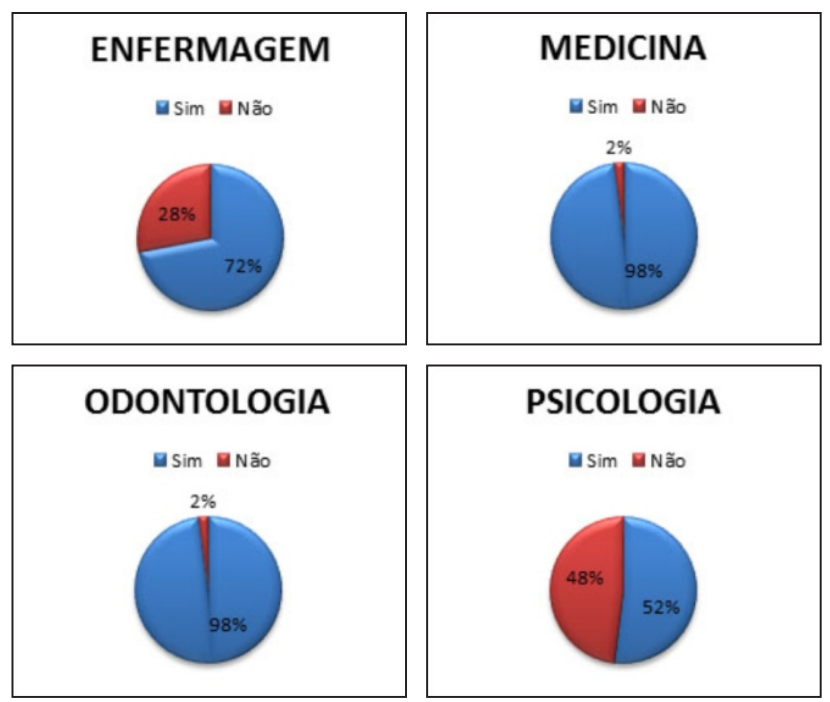

Figura 1 - Acadêmicos que conhecem ou ouviram falar da fissura labiopalatal.

Quando abordados sobre terem estudado sobre a anomalia durante o curso de graduação $98 \%$ dos alunos do curso de odontologia, 94\% dos alunos do curso de medicina, $52 \%$ do curso de enfermagem e $44 \%$ dos alunos do curso de psicologia relataram terem recebido informações durante o curso de graduação (Figura 2).
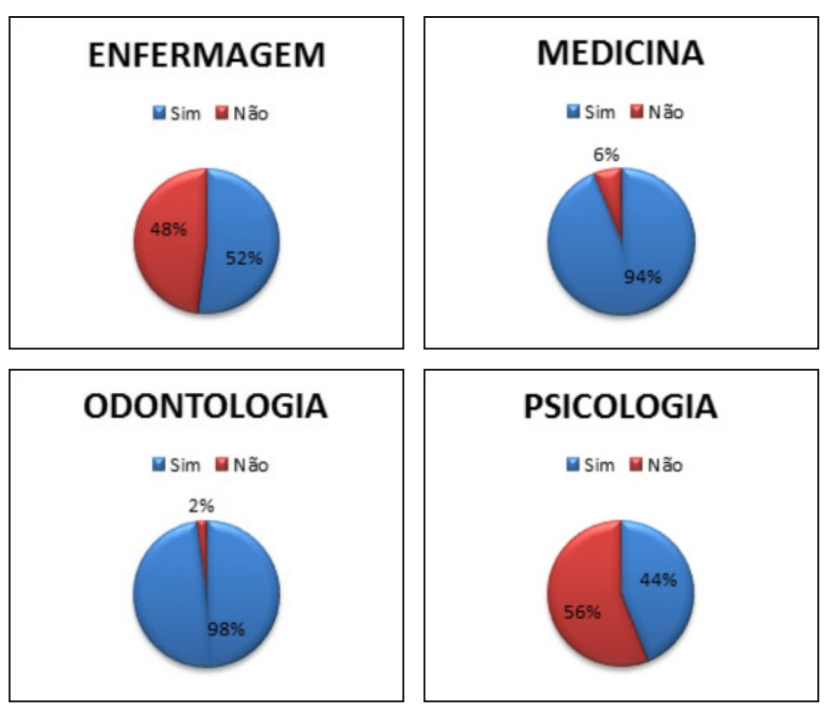

Figura 2 - Informações sobre fissura labiopalatal durante o curso de graduação.

O conhecimento da etiologia é fator de grande importância para o conhecimento da anomalia, quando os acadêmicos foram avaliados sobre o conhecimento da etiologia $14 \%$ dos alunos de odontologia foram capazes de responder a questão, contra $2 \%$ dos alunos de medicina e nenhum aluno de enfermagem e psicologia (Figura 3).
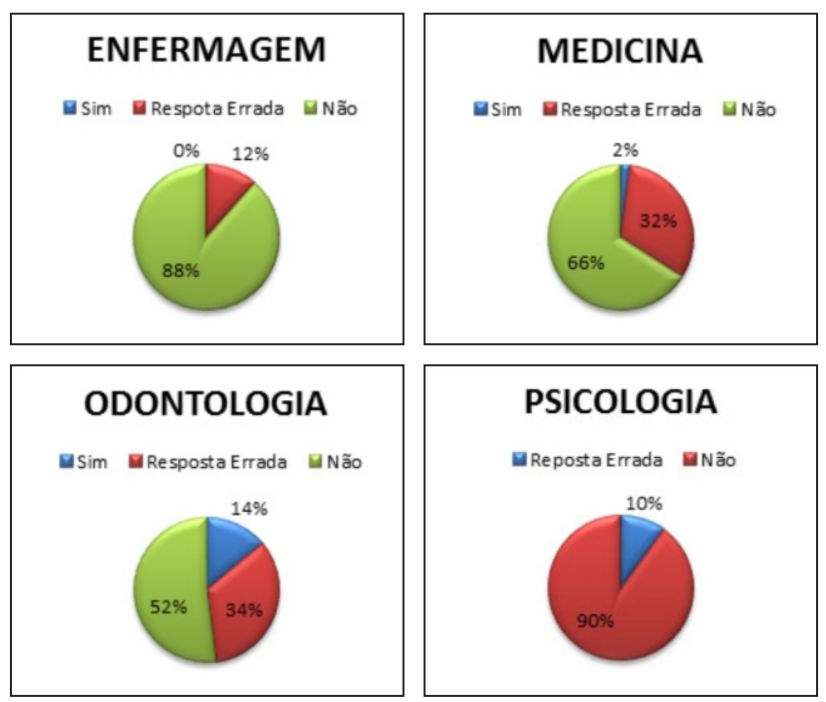

Figura 3 - Conhecimento da etiologia da fissura labiopalatina.

Para reabilitação de pacientes portadores é necessário que haja uma grande diversidade de profissionais em diferentes áreas de atuação, e a capacitação e segurança são fundamentais, quando questionados se o acadêmico se julgava capaz de atender um paciente com fissura labiopalatal apenas $20 \%$ dos alunos do curso de medicina, $46 \%$ dos alunos do curso de enfermagem, $44 \%$ dos alunos do curso de odontologia e $32 \%$ dos alunos de psicologia se declararam preparados para o atendimento (Figura 4). 

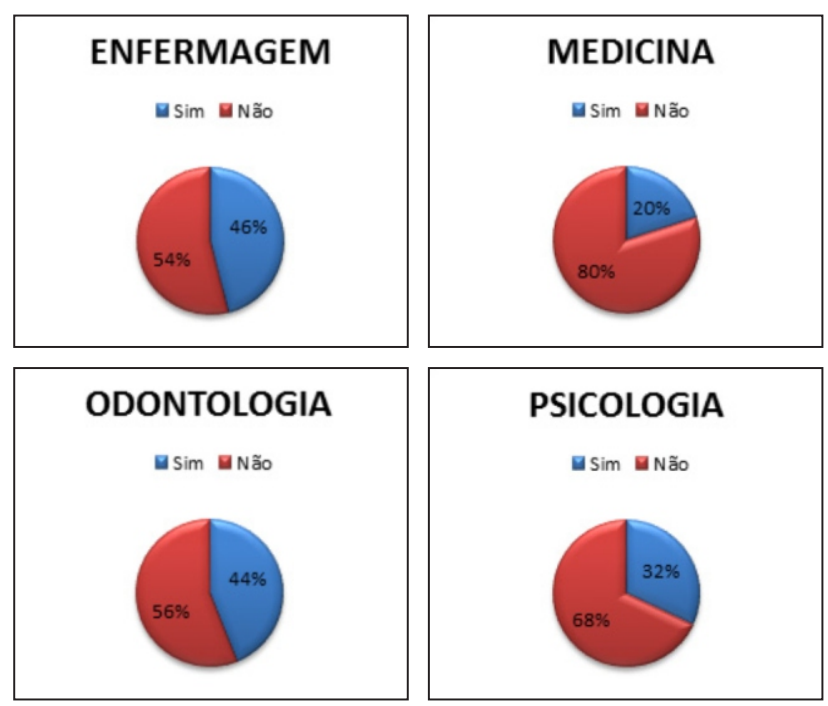

Figura 4 - Acadêmicos que se julgam aptos a atender pacientes portadores de fissura labiopalatal.

Outro fator importante é a capacidade de encaminhamento e o conhecimento de centros de referência para tratamento e reabilitação de pacientes portadores de fissuras labiais e/ou palatal, constatou-se que apenas $10 \%$ (medicina), 14\% (enfermagem), 14\% (odontologia) e $0 \%$ (psicologia) teriam condições de realizar o encaminhamento correto do paciente, sendo que apenas 10\% (medicina), $10 \%$ (enfermagem), 18\% (odontologia) e $0 \%$ (psicologia) conhecem de fato um centro de referência para encaminhamento e tratamento dos pacientes (Figura 5).
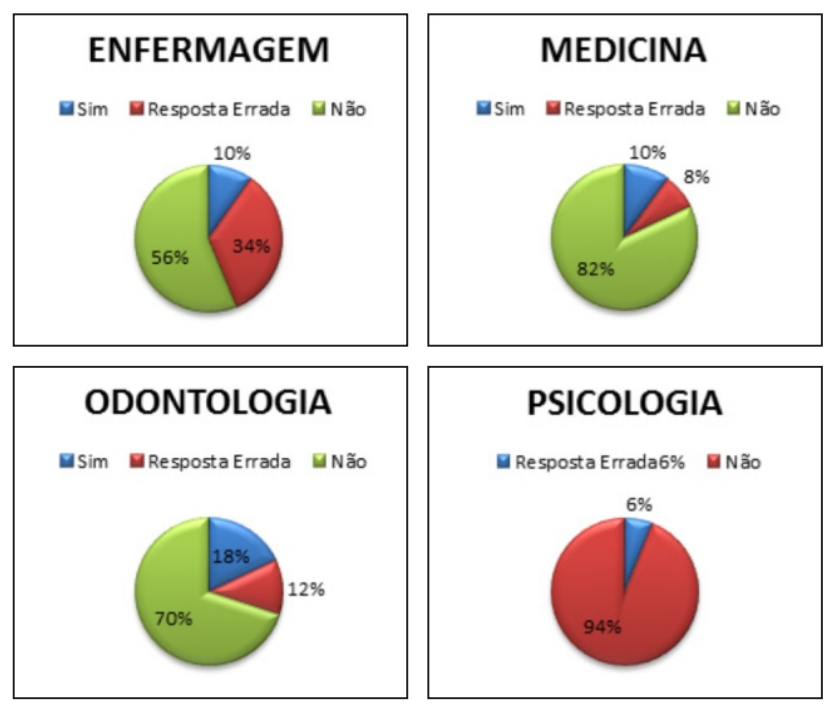

Figura 5 - Acadêmicos que conhecem algum centro de referência para tratamento de pacientes portadores de fissura de lábio e /ou palato.
Os acadêmicos foram inqueridos sobre os profissionais mais envolvidos no tratamento dos pacientes fissurados, sendo a odontologia área que mais foi pontuada, seguida da fonoaudiologia e medicina.
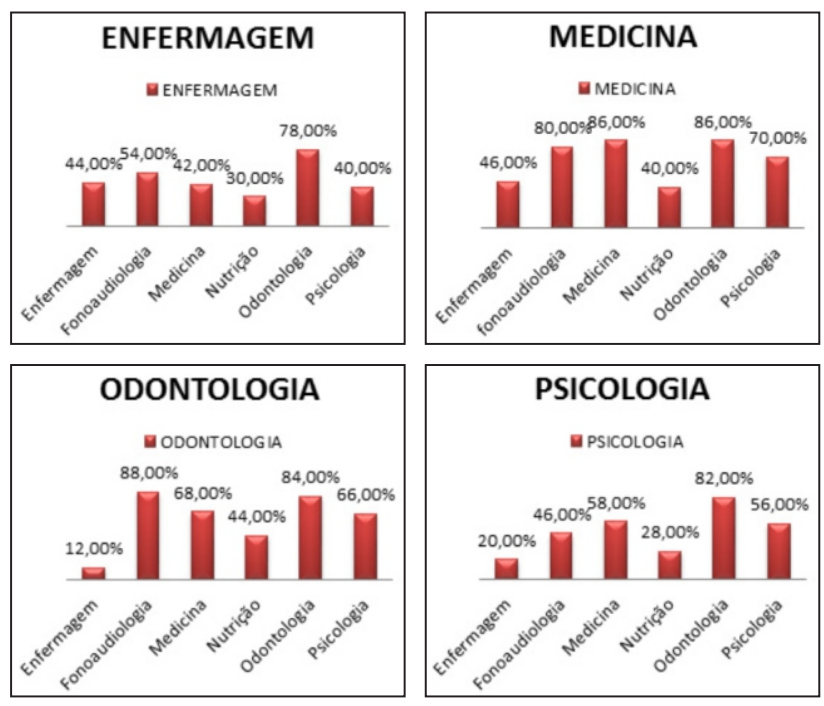

Figura 6 - Profissionais mais envolvidos no tratamento de pacientes fissurados.

\section{DISCUSSÃO}

O nascimento de uma criança portadora de fissura lábio palatal inaugura o início de uma longa jornada em busca da reabilitação. Tal tratamento envolve uma grande diversidade de profissionais e especialidades, compondo os três níveis de atenção pública brasileira, com o objetivo de se atingir uma adequada reabilitação estético funcional e reestabelecer a integração social e psicológica do indivíduol.

A abordagem precoce e adequada dos pacientes com fissura labiopalatal é fundamental para que haja aumento na qualidade de vida destes indivíduos6,23-24. O conhecimento sobre as fissuras labiopalatais durante o curso de graduação é extrema importância para a formação de profissionais mais capacitados e preparados para enfrentar uma grande diversidade de situações que ocorrem no atendimento público. O conhecimento sobre os tipos de fissura, sua classificação e a forma de atendimento irão influenciar as condutas adotadas pelos profissionais. A classificação e identificação das fissuras permite evidenciar as principais características de cada tipo de fissura, seja pelo aspecto físico, dificuldade de alimentação, higiene, controle periodontal ou mesmo pela falta ou posicionamento inadequado de dentes 10,25-27.

No Brasil, não há legislação específica sobre a composição da equipe de saúde ou seu modelo 
organizacional (multidisciplinar, interdisciplinar, transdisciplinar ou isolado). Portaria SAS / MS n. 62 da Secretaria de Atenção à Saúde e Ministério da Saúde estabelece que os hospitais registrados para a realização de procedimentos integrados de reabilitação estéticofuncional de pacientes com fissura lábio palatal devem oferecer as seguintes especialidades: cirurgia oralmaxilofacial, cirurgia plástica, odontologia (odontologia pediátrica, ortodontia, colocação de próteses, colocação de implantes), otorrinolaringologia, terapia fonoaudiológica, psicologia, serviço social, clínica médica, enfermagem, pediatria, anestesia, fisioterapia, nutrição e cuidados familiares1,28.

Os grandes centros de atendimento aos pacientes fissurados estão localizados principalmente na região sul e sudeste do país, e a grande distância dificulta o acesso e tratamento, principalmente por ser um tratamento prolongado e pelo baixo poder aquisitivo das pessoas 29 .

AFAMP mantém os cursos de Medicina, Enfermagem, Odontologia e Psicologia e prioriza a qualidade de ensino, informações básicas como a etiologia das fissuras são fornecidas ainda nos primeiros períodos da graduação, entretanto o pouco contato com grandes centros de atendimento e a escassez de casos clínicos, acabam prejudicando o aprendizado e a consolidação das informações prestadas.

Alguns resultados obtidos durante o questionário foram muito contraditórios como ocorreu nos questionários enviados aos cursos de enfermagem e psicologia, onde $52 \%$ e $56 \%$ respectivamente relataram ter tido informações na graduação e $48 \%$ e $44 \%$ respectivamente relataram não ter recebido nenhum tipo de informação durante a graduação, respostas assim geram preocupação e merecem ser melhor estudadas.

$\mathrm{O}$ tratamento multidisciplinar e o conhecimento sobre centros de referência onde o tratamento é oferecido representam porta de entrada para os pacientes e fonte de conhecimento para os profissionais, portanto a indicação e contato da instituição com tais centros são essenciais para uma melhor formação dos acadêmicos. Todos os alunos do estudo, independente do curso concordaram que o tratamento multidisciplinar é imprescindível e a grande maioria dos alunos concordam que as informações prestadas aos pais e o acompanhamento psicológico são fundamentais e devem fazer parte do tratamento do paciente fissurado30.

Os acadêmicos relataram que a especialidade mais envolvida no atendimento desses pacientes é a odontologia, quando na verdade a reabilitação é multiprofissional e depende do esforço conjunto de uma equipe multiprofissional altamente qualificada e preparada para as dificuldades diárias dos pacientes portadores das lesões, neste contexto é importante observar que os cursos de psicologia e enfermagem reduziram sua participação para
$56 \%$ e $44 \%$ respectivamente, priorizando outras áreas, fato que não ocorreu nos questionários realizados nos cursos de medicina e odontologia. Tendo-se o entendimento de que a reabilitação é multiprofissional, a participação de cada área é fundamental e determinante para os resultados, portanto os acadêmicos de psicologia e enfermagem desvalorizaram sua importância e subjugaram a importância das respectivas áreas no atendimento de pacientes portadores da anomalia.

A grande maioria dos alunos da FAMP relataram ter o desejo de participar de eventos, palestras ou outros cursos que fornecessem mais informações sobre o assunto, evidenciando o interesse dos alunos e a oportunidade da faculdade em promover eventos para a discussão e apresentação de casos e condutas reabilitadoras, fortalecendo o ensino e desta forma contribuindo ainda mais para o crescimento intelectual e prático dos alunos.

\section{CONCLUSÃO}

O conhecimento sobre os pacientes portadores de fissura labial e/ou palatal e a importância do tratamento multidisciplinar dos acadêmicos dos cursos de Medicina, Enfermagem, Odontologia e Psicologia da FAMP são superficiais e subjetivos. As informações sobre fisssuras são fornecidas ainda nos primeiros anos de graduação, sem que haja uma correlação prática, fato que associado à baixa frequência dos pacientes fissurados e a falta de parcerias com centros especializados culminam com a limitação dos atendimentos e consequentemente limitam também o conhecimento prático dos alunos da instituição.

\section{REFERÊNCIAS}

1. Lorenzzoni D, Carcereri DL, Lucks A. A importância do atendimento multiprofissional e interdisciplinar na reabilitação e promoção de saúde ao portador de fissura labiopalatal. Rev Odonto Cienc. 2010;25(2):198-203.

2. Montagnoli LC, Barbieri MA, Bettiol H, Marques IL, Souza L. Prejuízo no crescimento de crianças com diferentes tipos de fissura lábio-palatina nos 2 primeiros anos de idade. Um estudo transversal. J Pediatr (Rio J). 2005;81:461-5.

3. Silva DSF, Mauro LDL, Oliveira LB, Ardenchi TM, Bonecker M. Estudo descritivo de fissuras lábio-palatinas relacionadas a fatores individuais, sistêmicos e sociais. RGO. 2008;56(4):387-91.

4. Santos EC, Leite SGS, Santos SMP, Neves ZP, Passos XS, Silveira FFCF. Análise qualitativa do padrão alimentar de crianças portadoras de fissura de lábio e/ou palato atendidas em um hospital de Goiânia GO. Rev Inst Cienc Saude. 2011;29(3):183-5.

5. Mendes LGA, Lopes VLGS. Fenda de lábio e ou palato: recursos para alimentação antes da correção cirúrgica. Rev Cienc Med. 
2006;15(5):437-48.

6. Batista LRV, Triches TC, Moreira EAM. Desenvolvimento bucal e aleitamento materno em crianças com fissura labiopalatal. Rev Paul Pediatr. 2011;29(4):674-9.

7. Aguiar CR, Pinto LC, Nishiyama CK, Hussne RP. Avaliação da relação entre reabsorções dentárias e fissuras de lábio e/ou palato, e a sua prevalência em pacientes atendidos no Setor de Endodontia do Hospital de Reabilitação de Anomalias Craniofaciais. ROBRAC. 2014;23(65):89-93.

8. Dalben G, Costa B, Gomide MR. Características básicas do bebê 37 . portador de fissura labiopalatal: aspectos de interesse para o CD. Rev Assoc Paul Cir Dent. 2002;36:223-6.

9. Mutarai T, Ritthagol W, Hunsrisakhun J. Factors influencing early childhood caries of cleft lip and/ou palate children aged 18 to 36 months in southern Thailand. Cleft Palate Craniofac J. 2008;45:468-72.

10. Pedro RL, Tanurre PN, Antunes LAA, Costa M.C. Alterações do desenvolvimento dentário em pacientes portadores de fissura de lábio e/ ou palato: revisão de literatura. Rev Odontol Univ Sao Paulo. 2010;22(1):65-9.

11. Mendes M, Silveira MM, Costa FS, Schardosim LR. Avaliação da percepção e da experiência do cirurgiões-dentistas de rede municipal de Pelotas/RS no atendimento dos portadores de fissuras labiopalatais. Rev Fac Odontol Univ Passo Fundo. 2012;17(2):196-200.

12. Froes Filho RR. Tratamento de pacientes portadores de fissuras 8 . labiopalatais. Rev Assoc Odontol Norte do Paraná. 2003;17:26-7.

13. Tannure PN, Molitermo LFM. Fissura palatina: apresentação de um caso clínico. Rev Odontol UNESP. 2007;36(4):341-5.

13. Rafacho MB, Tavano LD, Motti TFG. As perguntas mais frequentes dos pais de crianças com fissura labiopalatina. Rev Bras Med Mod. 2015;51(3):106-10.

14. Vanz AP, Ribeiro NRR. Escutando as mães de portadores de fissuras orais. Rev Esc Enferm USP. 2010;45(3):596-602.

15. Alves A, Carrara CFC, Costa B. Influência da conduta dos pais sobre o comportamento de crianças com fissura de lábio e/ou palato diante do tratamento odontológico realizado no Hospital de Reabilitação de Anomalias Craniofaciais. Pediatr Mod. 2008;44(3):88-94.

16. Brasil FR, Tavano LA. Escolha de parceiros afetivos: influência das sequelas de fissura labiopalatal. Paideia. 2007;17(38):375-87.

17. Vrijheid H, Dolk H, Stone D, Abramsky L, Alberman E, Scott JE. Socioeconomic inequalities in risk of congenital anomaly. Arch Dis Child. 2000;82:349-52.

18. Jayasekera T, Hall R, Lopacki S. Tratamento das fissuras labiopalatais. In: Cameron A, Widmer R. Manual de odontopediatria. São Paulo: Ed. Santos; 2001. p. 289-305.

19. Graciano MIG, Tavano LDA, Bachega MI. Aspectos psicossociais da reabilitação. In: Trindade IEK, Silva Filho OG da. Fissuras labiopalatinas: uma abordagem interdisciplinar. São Paulo: Ed. Santos; 2007. p. 311-33.

20. Silva EB, Fúria CL, Di Ninno CQ. Aleitamento materno em recémnascidos portadores de fissura labiopalatina: dificuldades e métodos utilizados. Rev CEFAC. 2005;7:21-8.

21. Schardosim LR, Nogueira DA, Bosco VL, Pereima MJL. Bebês portadores de fissura labiopalatal: satisfação dos pais com as orientações recebidas dos profissionais. Rev Iberoam
Odontopediatria Odontol Bebe. 2004;40:568-73.

22. Amaral CER, Kuczynski E, Aloso N. Qualidade de vida de crianças com fissura labiopalatina: análise crítica dos instrumentos de mensuração. Rev Bras Cir Plast. 2011;26(4):639-44.

23. Zhou M, Liu Z.X, Wang P.L, Liu C. Quality of life and its influential factors of children and adolescents with congenital cleft lip and palate. Shanghai Kou Qiang Yi Xue. 2016;25(1):63-7.

24. Cymrot M, Sales FCD, Teixeira FAA, Teixeira Junior FAA, Teixeira GSB, Cunha Filho JFC, et al. Prevalência dos tipos de fissura em pacientes com fissura labiopalatais atendidos em um Hospital Pediátrico do Nordeste brasileiro. Rev Bras Cir Plast. 2010;25(4):648-51.

25. Menezes, L.M.; Rizzatto, S.M.D.; Aazaredo, F.; Vargas, D.A. Características e distribuição de anomalias dentarias em uma população brasileira de portadores de fissura lábio-palatais. Rev Odonto Cienc. 2010;25(2):137-41.

26. Muncinelli EAG, Oliveira GHC, Esper LA, Almeida ALPF. Aspectos periodontais em pacientes com fissuras labiopalatinas. Rev PerioNews. 2012;6(4):359-63.

27. Brasil. Ministério da Saúde. Portaria SAS/MS n. 62, 19 de abril 23. de 1994. Normaliza cadastramento de hospitais que realizem procedimentos integrados para reabilitação estético-funcional dos portadores de máformação lábio-palatal para o Sistema Único de Saúde. Diário Oficial da União. 1994:3.

28. Feitas JAS, Dalben GS, Santamaria M Jr, Freitas PZ. Current data on the characterization of oral clefts in Brazil. Braz Oral Res 2004;18:128-33.

29. Maggi A, Scopel JB. Atendimento aos portadores de fissuras labiais e/ou palatais: características de um serviço. Rev Aletheia. 2011;6(34):175-86. 